\title{
Inventory control systems for stochastic lead time demand
}

\author{
Mustafid $^{1,2, *}$, Dwi Ispriyanti ${ }^{3}$, Sugito $^{4}$, and Diah Safitri ${ }^{5}$ \\ ${ }^{1}$ Master Program of Information Systems, School of Postgraduate Studies, Diponegoro University, Semarang - Indonesia \\ $2,3,4,5$ Department of Statistics, Faculty of Sains and Mathematic, Diponegoro University, Semarang - Indonesia
}

\begin{abstract}
Product inventory control can be used to provide information related to processes of production and distribution products based on lead time and product demand from consumers. The research aims to design product inventory control systems for stochastic lead time demand, and using exponential smoothing to make lead time demand prediction. The design of the control system uses the probability method based on the estimated number of product demand from the market or consumer. This research provides theoretical framework for control systems of products that can determine the size of inventory parameters in supply chain management, that is reorder point and fixed order quantity, so it can manage the production amount and supply of products according to market or consumer demand. The benefits of a product control systems in the inventory management are to provide the necessary inventory allocation information in making decisions to determine the amount of production and distribution of products to consumers.
\end{abstract}

Keywords: Probabilistic method; inventory control systems; stochastic lead time demand

\section{Introduction}

In highly competitive markets, all manufacturers seek to improve the quality of their industrial products, by reducing product and service costs, and to shorten product delivery and distribution time to consumers [1]. In the era of quality and business competition is very tight, the life cycle of clothing products to the consumer demands with a shorter time and has an element of uncertainty. This can lead to a high risk in the clothing supply chain management industry [2].

Currently, the clothing industry market changes increasingly dynamic and complex, and must face market changes every day. This should be a management action to be able to predict and prepare the business for each day that changes [3]. Dynamic changes in the clothing industry must be followed by inventory management that can anticipate business processes that require various stages, and each stage is accompanied by an element of uncertainty caused by uncertain market demand.

In order for the clothing industry to succeed in a highly competitive business environment, clothing industry must be able to run supply chain inventory management in order to improve supply chain performance continuously [4]. The inventory control model (ICM) is part of the supply chain inventory management that plays an important role in controlling the company resources in all links by paying attention to the quality of each supplier link to the customer $[5,6]$. Many companies are faced with a rigorous, competitive environment and emerging uncertainties as a result of information technology innovation and changing customer needs. Inventory control (IC) can be used for consistent and sustainable performance measurement of each link in the supply chain that makes a key role in the success of a company to achieve a business target goal with a certain profitability [7].

The ICM must be adaptive to adapt to changes in market or consumer demand. As an example of a higher level of consumer demand from inventory will have an impact on the aspect of customer satisfaction to get the product according to the desired time. In this case, ICM aims to overcome the existence of deficiency or excess product in accordance with production capacity and inventory [8].

Probabilistic and statistical approaches for inventory control systems (ICS) in the clothing industry focus on two variables, that is variables of demand and lead time. Since both variables are stochastic, modeling the IC using the probability distribution assumption of both variables.

The use of assumptions for daily demand has a normal distribution can yield more suitable results, but the assumption of a normal distribution of demand data is often not appropriate, especially if demand per unit of time or lead time are fluctuating or has a large variance. The compound distribution of demand during the lead time is use by the distribution of demand and the distribution of lead times. The probability distribution of the combined variables can be normal or other

\footnotetext{
* Corresponding author: mustafid55@gmail.com
} 
distributions according to the data characteristics of daily demand and lead time periodically.

The research aims to apply ICS on the predicted lead time demand (LTD) using probabilistic and statistics approach. Prediction method in the research using exponential smoothing approach. The ICS can be used to determine the numbers of safety stock (SS) and reorder point (ROP) for planning of product inventory renewal according to consumer demand.

\section{Research methods}

The method of research is a implementation of probability and statistics for industry and business. The study of IC with deterministic demand data has been done by [6] and [9]. The supply chain system framework is based on information flow in the form of product demand, and material flow of products has been developed by [7]. The concept of supply chain system can be adjusted to be the basis in modeling inventory control product.

The research designs ICS that can be used to analyze SS and ROP based on the combined of demand and lead time stochasticlly. The material studied in this research includes the use of probabilistic and statistical approaches in ICM. In this case study, the data used were not normally distributed, so the exponential smoothing approach use for transformation into normal distributed data, and also as a prediction model in the ICM based on previous actual data of demand.

The variables in this research use the number of daily demand and lead times, taking a case study in the clothing industry. Data of daily demand in the form of historical data from daily demand. The observational data a sample were taken for 105 days with 8 lead times from April to June 2016. Daily demand data and lead times are define as stochastic variables.

The variability of demand and lead time are the basic for making theoretical development of theory and its application in IC. SS determination uses a combined of daily demand and lead time into variables of LTD. In statistical theory, combined variables are called compound variables. The LTD is a random variable, in which those distribution is determined by the combined of the distribution of demand variable and lead variable.

Data of demand from consumers or transactions with consumers in retail are monitored through demand data on supply chains sent by retail every day. The demand data is input to the system, then processed to produce the output of estimation of SS and ROP as the basis for performing the amount of stock order that is useful for arranging product quantities and product distribution according to the number of demand.

\section{Stochastic lead time demand}

The ICM is designed using variable of LTD based on demand on the lead time from consumers or retail. Let $D$ be a srandom variable in the form of a daily demand in a periodic lead time $L$. Daily demand $(D)$ and lead time $(L)$ are stochastic random variables. Daily demand and lead time are assumed to be random variables that are mutually independent and distributed identically. The random variable of demand $\mathrm{D}$ is also assumed to be independent with the lead time L. LTD $X$ at time $L$ is expressed by:

$$
X=D_{1}+D_{1}+\cdots+D_{L}
$$

Suppose mean and variance of $\mathrm{D}$ and $\mathrm{L}$ are respectively expressed by:

$$
\begin{array}{lll}
\mathrm{E}(D)=\mu_{D} & \text { dan } & \operatorname{Var}(D)=\sigma_{D}^{2} \\
\mathrm{E}(L)=\mu_{L} & \text { dan } & \operatorname{Var}(L)=\sigma_{L}^{2}
\end{array}
$$

The random variables of $\mathrm{D}$ and $\mathrm{L}$ are can be estimated using observation data. The mean values of (2) and (3) can be estimated by data observations:

$$
\begin{aligned}
& \bar{D}=\frac{1}{N} \sum_{i}^{N} D_{t} \\
& \bar{L}=\frac{1}{k} \Sigma_{i}^{k} L_{t}
\end{aligned}
$$

Similarly, the variance values of (2) and (3) can be estimated by:

$$
\begin{aligned}
& S_{D}^{2}=\frac{1}{N-1} \sum_{t=1}^{N}\left(D_{i}-\bar{D}\right)^{2} \\
& S_{i}^{2}=\frac{1}{k-1} \sum_{t=1}^{k}\left(L_{i}-\bar{L}\right)^{2}
\end{aligned}
$$

whrere $D_{1}, D_{2}, \ldots, D_{N}$ are data of demand with $N=L_{1}$ $+L_{2}+\cdots+L_{k}, L_{\mathrm{i}}$ is lead time in i period, $i=1, \ldots, k$.

Using the unbiased properties of variable of demand $\mathrm{D}$ and lead time L, then by (5), (6), (7) and (8) obtained:

$$
\begin{array}{ll}
\mathrm{E}(\bar{D})=\mu_{D} & \text { dan } \mathrm{E}\left(S_{D}^{2}\right)=\sigma_{D}^{2} \\
\mathrm{E}(\bar{L})=\mu_{L} & \text { dan } \mathrm{E}\left(S_{V}^{2}\right)=\sigma_{L}^{2}
\end{array}
$$

Therefore, the estimation for the mean and variance of demand and lead time variables can be calculated with the mean and variance values of observational data (samples) with formulas (5), (6), (7) and (8).

The variable $X$ in equation (1) can be define as a compound random variable, and mean and variance of $X$ can be derived by:

$$
\begin{aligned}
\mathrm{E}(X) & =\mathrm{E}\left[\sum_{l=1}^{L} D_{i}\right]=\mu_{L} \mu_{D} \\
\operatorname{Var}(X) & =\mathrm{E}\left[X_{L}-\mathrm{E}\left(X_{L}\right)\right]^{2} \\
& =\mu_{L} \sigma_{D}^{2}+\sigma_{L}^{2} \mu_{D}^{2}
\end{aligned}
$$

In certain cases, if $\mathrm{D}$ and $\mathrm{L}$ have a normal distribution, then distribution of $X$ can be determined as a normal distribution. For example, $D_{i}$ is random variable having a normal distribution with mean $\mu_{D}$ and variance $\sigma_{D}^{2}$, , it can be proved that $X$ as a new random variable following the normal distribution with mean $\mu_{L} \mu_{D}$ and variance $\mu_{L} \sigma_{D}^{2}+\sigma_{L}^{2} \mu_{D}^{2}$.

\section{Lead time demand prediction}

The prediction of the number of demand in a certain period is based on the forecast data from the previous demand. The prediction model for next demand use exponential smoothing approach of the demand during period T [10] with illustration as in Fig. 1 and Fig 2.

Let $D_{t}$ is the daily demand as stochastic process at time t. Predicted daily demand D for the next one (Fig 1) is calculated based on the previous time period $\mathrm{T}$ using exponential smoothing with the formula:

$$
\widehat{D}_{t+1}=\alpha D_{t}+(1-\alpha) \sum_{l=t-1-T}^{t-1} D_{l} / \mathrm{T}
$$

where $\widehat{D}_{t+1}$ is the demand prediction for time $t+1, D_{t}$ is the actual demand at time $t$, and $\alpha$ is the parameter which is the weight of the previous demand. The weight value 
of $\alpha$ is derived for data is normal distribution with a low error.

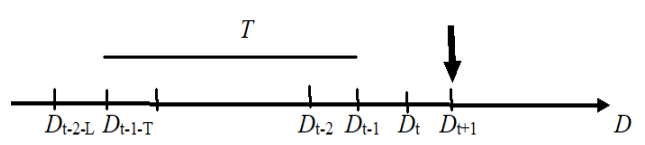

Fig. 1. Daily demand prediction

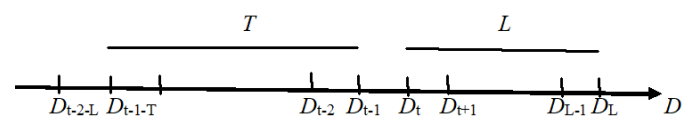

Fig. 2. Demand prediction for next lead time

Furthermore, using (11) can be calculate the prediction of demand for period $t$ to lead time $\mathrm{L}$, (or $\mathrm{t}_{\mathrm{j}}+$ $\mathrm{L}_{\mathrm{j}}$ ) by formula:

$$
\widehat{D}_{t_{j}}=\Sigma_{l=T}^{t_{j}+L_{j}} \widehat{D}_{l}
$$

Based on (12), we can define prediction of all daily demand with $\mathrm{k}$ lead time by:

$$
\AA=\sum_{j=1}^{k} \widehat{D}_{t_{j}}
$$

The value of mean and variance from $\hat{X}$ calculated in the same way by the formula (9) and (10).

The computational results for parameters of the demand and lead time using the formula (5), (6), (7), (8) and (9) are given in Table 1. The profile of actual daily demand data and the smoothing data predicted with exponential smoothing are given in Fig, 3. Based on the profile in Fig 3, show that variable of demand and lead time are probabilistic. Using the formula (12) and (13), we can calculate the value of total and average of LTD prediction based on all daily demand and all lead time as a basis for determining ROP and ROP,

Table 1. Parameter of lead time and LTD

\begin{tabular}{|l|c|c|c|}
\hline Parameter & $\begin{array}{c}\text { Lead } \\
\text { time }\end{array}$ & $\begin{array}{c}\text { Demand } \\
\text { prediction }\end{array}$ & $\begin{array}{c}\text { LTD } \\
\text { prediction }\end{array}$ \\
\hline Average & 13 & 91 & 1183 \\
\hline Variance & 6.98 & 806.50 & 68285.87 \\
\hline $\begin{array}{l}\text { Deviation } \\
\text { Standar }\end{array}$ & 2.64 & 28,01 & 261.32 \\
\hline
\end{tabular}

Data of demand derived from the observational data satisfies the assumption of mutually independent and distributed identical. The probability approach is used to find the probability distribution of LTD. To prove that the data of demand and lead time have a normal distribution is used the Kolmogorov-Smirnov test. The result of normal distribution hypothesis test for actual demand data gives $p$-value $=0,026$, which states that actual data of demand is not normally distributed for $\alpha=$ 0,05 . Therefore, it is necessary to refine the data with exponential smoothing method using formula (11).

The smoothing of demand data with exponential smoothing (11) is given in Fig.3, and based on Kolmogorov-Smirnov test for the smoothing of demand gives $\mathrm{p}$-value $=0,200$. Using $\alpha=0.05$, and since $\mathrm{p}$-value $=0.200>\alpha=0.05$, thus accepting the hypothesis that the data of smoothing demand/ predicted of demand is normally distributed. Likewise for the observation data lead time, obtained $p$-value $=0.200$, so that lead time is also normally distributed.

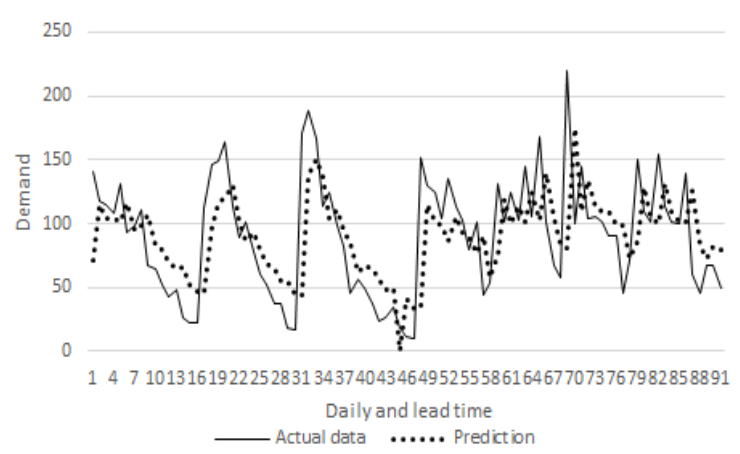

Fig. 3. Chart of actual and prediction demand

The prediction model with exponential smoothing (11) can be used for the smoothing model as a transformation model for normal distribution and as a prediction model. The prediction accuracy can be determined by the MAPE (Mean Absolute Percentage Error) method, with the formula:

$$
\text { MAPE }=\frac{1}{N} \sum_{t=1}^{N}\left|\frac{x_{t}-\hat{x}_{t}}{x_{t}}\right| \times 100 \%
$$

where $\mathrm{N}$ is the number of daily demand data used for the prediction model. The computational results of the predicted data in chart form are given in Fig. 3. Furthermore, the computational results of MAPE show that for the prediction of demand obtained MAPE $=45 \%$ (reasonable), with for predicted result for LTD obtained MAPE $=6 \%$ (high accuracy). Although MAPE for data demand data prediction is quite large, but for data prediction of LTD is very small.

\section{Inventory control systems}

ICS is designed using ICM within the supply chain information system (SCIS) framework with daily demand and lead times as the input of systems. Daily demand and lead time is used to make predictions of product needs as materials to plan the number of products to be produced. The prediction model use exponential smoothing method with equation (11).

The design of ICS for the clothing industry are given in Fig. 4, with system inputs in the form of daily demand and lead times (Fig 3). Procedures in access to data input for the system, the input system in the form of demand from consumers, initially accommodated in retail, so accessed into the database system conducted by administrators and retail. The system database is designed as an integrated database within ICS.

Fig. 4 shows that the consumer or retail demand data is the basis for making predictions of the number of products produced. Predicted product quantities and product inventory estimates are determined on the basis of SS and ROP as parameters in IC. Web administrators have a master data entry facility that is used to estimate the management of product stock for raw material planning required in producing a number of planned 
products. The retailer sends the demand and sales in accordance with the product code and the amount sold. This activity is conducted by using mobile devices such as SMS / WA every day.

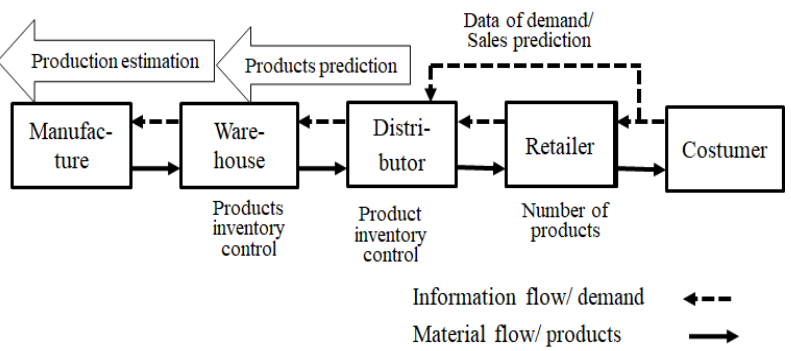

Fig. 4. Inventory control systems

The ICS is defined as a tool for the management and control of supply, storage, distribution, and recording of products to maintain adequate stock quantities in accordance with consumer demand or needs. With this control system is expected to generate maximum profits with minimal inventory investment without reducing the level of customer satisfaction or the level of demand or order filling. Three parameters used for control inventory:

a. Safety stock (SS),

b. Reoder point (ROP),

c. Fixed order quality (Q).

Probability approach with normal distribution is used to make ICM with input data of demand and lead time in the form of random and stochastic variable. To state that the stochastic process or random sample data satisfy the assumption of normal distribution will be use by Kolmogorov-Smirnov test. With normal distribution function it can be used to determine $\mathrm{SS}$ and ROP as given in Fig. 5.

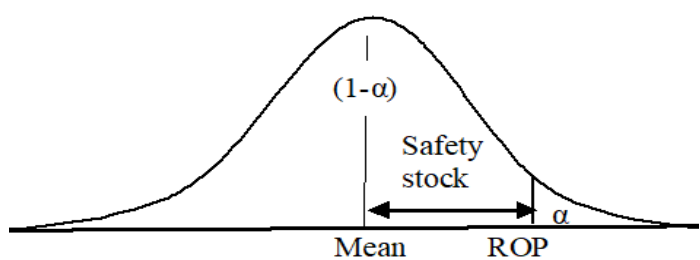

Fig. 5. Curve of normal distribution for inventory control

Using the properties of the normal probability for random variable $X$, then expecting $X \leq \mathrm{ROP}$, it can be determined the probability that LTD is less than ROP with the least probability of $1-\alpha$ :

$$
\mathrm{P}(X \leq \mathrm{ROP}) \geq 1-\alpha
$$

as given in Fig. 5. If using $\alpha=0.05$ or $5 \%$, then probability for LTD is less than ROP given in the formula:

$$
\mathrm{P}(X \leq \mathrm{ROP}) \geq 1-0,05=0,95
$$

Therefore, the level of confidence that inventory for demand is always over $95 \%$. Using the properties of the confidence interval on the normal distribution curve (Fig. 5), it is obtained that

$$
\begin{aligned}
\mathrm{ROP} & =\mathrm{E}(X)+z_{a} \sqrt{\operatorname{Var}(X)} \\
& =\mu_{L} \mu_{D}+z_{\alpha} \sqrt{\mu_{L} \sigma_{D}^{2}+\sigma_{L}^{2} \mu_{D}^{2}}
\end{aligned}
$$

$$
\mathrm{SS}=z_{a} \sqrt{\mu_{L} \sigma_{D}^{2}+\sigma_{L}^{2} \mu_{D}^{2}}
$$

Furthermore, using data input of demand and lead time, and using formula (16) with $\alpha=0,05, Z_{\alpha}=1,65$, obtained:

$$
\begin{aligned}
\mathrm{SS} & =z_{\alpha} \sqrt{\mu_{L} \sigma_{D}^{2}+\sigma_{L}^{2} \mu_{D}^{2}} \\
& =431 .
\end{aligned}
$$

and by formula (15), obtained:

$$
\begin{aligned}
\mathrm{ROP} & =\mu_{L} \mu_{D}+Z_{\alpha} \sqrt{\mu_{L} \sigma_{D}^{2}+\sigma_{L}^{2} \mu_{D}^{2}} \\
& =1632 .
\end{aligned}
$$

The result of computing for parameter of the ICS is visualized with the output of a normal distribution curve showing the mean, SS and ROP as given in Fig. 6.

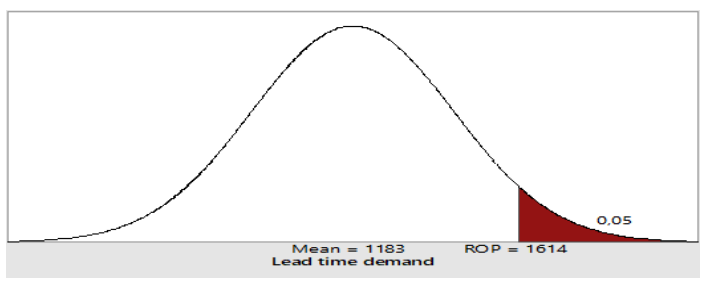

Fig. 6. Output for SS and ROP

In IC on the clothing supply chain industry, stock supply monitoring on the supply chain is based on checking on the current stock status. Current stock is calculated by:

Current stock $=$ initial stock - total demand

Based on demand data, then at the end of the lead time can be done the latest stock calculation (end), that is the initial stock at the beginning of lead time minus the number of LTD. Furthermore, the latest stock results compared to SS.

Procurement of stocks is carried out on retail requirements for products based on the requirement that:

a. No inventory is procured, if the current inventory number is greater than the number of SS of the product : IP - LTD $>$ SS.

b. If the current inventory number is smaller than the number of SS inventory, a procurement demand will be made, with quantity demand $\mathrm{Q}$ is determined by formula:

$$
Q=S S-I P+L T D
$$

where IP is the current stock position, LTD is the number of LTD, SS is the SS of the product, whereas Q is the number of product demand. Using the formula (17), the number of stocks delivery is as follows:

$$
\begin{aligned}
Q & =S S-I P+L T D \\
& =431-266+1318 \\
& =1483 \mathrm{pcs}
\end{aligned}
$$

Stock monitoring is done by looking at the latest stock system output at lead times compared to SS. If the current stock is larger than the SS, then the stock is in a safety condition, meaning no stock renewal is made. On the contrary, if current stock is smaller than SS, then stock is in watch condition, that means stock updating must be done soon, with minimum stock is ROP. In this case research, for the last lead time it appears that the current stock is 266 pcs. By comparing the $\mathrm{SS}=431$, the current stock is smaller than SS. So as soon as the stock renewal is equal to $\mathrm{Q}=1483$ for the next lead time. 
The fulfill assumptions of normal distribution for demand data as a condition of using SS formula (16), while stocks are safety determinant factor for calculating stock renewal. The use of the probability method for lead time demend modeling with a more accurate estimation of inventory parameters should take into account all available demand data within a given time period of a stochastically. In the traditional approach, demand parameters are estimated taking into consideration only with demand during the lead period. The length of lead time can be either constant or deterministic, but the beginning of the refill process is not known before, so simply using the demand during the lead time alone can cause a very bad estimate.

Because demand is stochastic, the number of demand often changes. This has an impact on SS, ie that SS tend to be low when demand intensity is low, and sometimes high if demand intensity is high. To overcome this, the determination of $\mathrm{SS}$ is expected to overcome the deviation (demand) on a particular day that often occurs.

Many IC research by establishing a safe stock use the assumption that demand and lead times are deterministic $[10,11]$. Similarly, the number of demand for any given time period is not known in advance, either the number of demand or the time of the demand. The problem has an impact on the results of the analysis that is not necessarily realistic. Other obstacles in IC, product delivery time can be uneven and uncertain, for several reasons, such as the amount of demand with erratic time, quality and transportation problems.

This research provides a new implementation of theory on ICS designed within the framework of SCIS with probability approach. This result is consistent with the results of previous research. For example [12] which states that studying the behavior of demand and lead time is very important to achieve a useful system representation in order to make the right decision.

\section{Conclusion}

Inventory control systems is designed in the SCIS framework using input demand and lead time. As a case study, Inventory control systems can be used to determine the number of safety stock and reorder point of lead time demand according to market or consumer demand. Determination of safety stock and reorder point using normal distribution curve.

By using properties on the normal distribution curve, we can calculate the parameters in inventory control management, ie safety stock and reorder point with significance level $1-\alpha$. The parameter of inventory control are used to determine an approximate minimum amount to perform a stock renewal.

\section{References}

1. D.M. Gligor, C.L. Esmark, M.C. Holcomb. Journal of Operations Management 33, 1 (2015).

2. A. Agarwal, R. Shankar, M.K. Tiwari. Industrial Marketing Management 36 (2007).

3. B.S. Sahay, J. Ranjan. Information Management \& Computer Security 16, 1 (2008).

4. F. Jie, K. A. Parton, Mustafid. International Journal of Logistics Research and Applications 19, 4 (2016).

5. D. A. Efrilianda, Mustafid, I. Rizal..IEEE Xplore (2018).

6. N. Aini, Mustafid, R. Kusumaningrum. IEEE Xplore (2018).

7. Mustafid, A. S. Karimariza, F. Jie. International Journal of Agile Systems and Management 11, 1 (2018).

8. S. Pazhani, J. A. Ventur, A. Mendoza. Applied Mathematical Modelling 40, 1 (2016),

9. A. D. Sabila, Mustafid, S. Suryono. In E3S Web of Conferences 31 (2018).

10. M. Rawat, T. Altiok. International Journal of Production Research (2008)

11. D. Prak, R. Teunter, A. Syntetos. European Journal of Operational Research 256, 2 (2016).

12. R.F. Roldán, R. Basagoiti, L.C. Coelho. J. Appl. Log., in press (2016). 